\title{
Modeling of critical systems implementing negative events using dual Petri nets
}

\author{
Marina A. Ganzhur*, Aleksei P. Ganzhur, and Olga V. Smirnova \\ Don State Technical University, 344000 Rostov-on-Don Gagarina 1, Russian Federation
}

\begin{abstract}
This work is devoted to solving the problem of modeling critical systems based on the use of modified Petri nets. The dual (binary) Petri net one of the modifications, which allows us to view inversing events at the same time, solving the problem with the possibility of deadlocks. Construction of schemes using fuzzy logic makes it possible to calculate the values of linguistic variables obtained knowledge. Petri dual network allows you to organize the exclusion of negative events by introducing additional links. In accordance with the rules, it is possible to construct a dual fuzzy Petri net, which involves the use of maximum and minimum transitions or appropriate logical calculations of conjunctions and disjunctions. Transition from classical Petri nets to dual fuzzy nets, realizes fuzzy knowledge of logical deriving that gives the chance in construction of expert systems with fuzzy logic solving a problem of data analysis.
\end{abstract}

\section{Introduction}

The problems of ensuring the safety of the functioning of critical systems lead to the need to develop the methods for modeling the negative events that arise in these negative events. Negative events are events that a person assesses as unfavorable. For example, events leading to dead ends and traps that exclude further functioning of the systems with asynchronous architecture and parallel functioning can be considered as negative events for abovementioned systems. Petri nets $[1,2]$ can be used to represent such systems. Petri nets allow developing the sufficiently complete models of critical systems, but herewith the Petri nets are not used to represent negative events that arise during the operation. The reason for this is the limitation, known as the inability to check for a zero mark of a particular position; this property is known as a «check for zero» [3]. In order to eliminate abovementioned disadvantage, it is possible to use binary Petri nets with alternative marking of positions [4], with their proven properties that they have the possibility to perform the checking for zero marking [5]. Such Petri nets, also called as dual Petri nets [6], differ from known nets by introducing the additional inverse positions for each direct position. Simultaneously, taking into account direct and inverse positions in the modeling of systems by using the dual Petri net, we can obtain knowledge about the states of the

\footnotetext{
* Corresponding author: mganzhur@yandex.ru
} 
system that create preconditions for the occurrence of negative events and propose the structural solutions for their elimination.

\section{Dual Petri net}

The dual Petri net is defined by the aggregate of sets (1):

$$
\mathrm{D}=\{\mathrm{P}, \mathrm{T}, \mathrm{I}, \mathrm{O}\}
$$

where: P - finite aggregate of positions divided into two sub-aggregates having the same numbering of positions. One sub-aggregate contains the so-called «direct positions» P1, the other, P0, contains «inverse positions» with the same numbers. Each number corresponds to binary positions: pi1 - direct position and pi0 - inverse position;

$\mathrm{T}$ - finite aggregate of transitions,

$\mathrm{P} \cap \mathrm{T}=\varnothing$;

$\mathrm{I}: \mathrm{T} \rightarrow \mathrm{P}-$ input function;

$\mathrm{O}: \mathrm{T} \rightarrow \mathrm{P}-$ output function.

The marked dual Petri net is the five (2):

$$
\mathrm{D} \mu=\{\mathrm{P}, \mathrm{T}, \mathrm{I}, \mathrm{O}, \mu\},
$$

where: $\mu$ - integer vector (3)

$$
\mu=(\mu 1(\mathrm{p} 11), \mu 2(\mathrm{p} 10), \ldots \mu \mathrm{k}-1(\mathrm{pn} 1), \mu \mathrm{k}(\mathrm{pn} 0)),
$$

herewith, $\mu$ (pi1) $\neq \mu$ (pi0).

In a binary position with the $\langle\mathrm{j}\rangle$ number, the mark is contained in only one position, either a direct position or an inverse position.

\subsection{Resources modeling}

Let us consider the dual Petri net, modeling the distribution of resources with the possibility of the occurrence of deadlocks.

Initial marking of the dual Petri net Fig. 1.

$$
\mathrm{P} 11=1, \mathrm{P} 20=1, \mathrm{P} 30=1, \mathrm{P} 41=1, \mathrm{P} 51=1, \mathrm{P} 61=1, \mathrm{P} 70=1, \mathrm{P} 80=1 .
$$

Alternative positions have opposite marks corresponding to 0 .

The occurrence of events consisting in the implementation of the actions by $\mathrm{t} 1$ and $\mathrm{t} 4$ transitions occurs as a result of performing the $\mathrm{P} 11=1, \mathrm{P} 20=1, \mathrm{P} 41=1$ preconditions for $\mathrm{t} 1$ transition and $\mathrm{P} 51=1, \mathrm{P} 61=1, \mathrm{P} 70=1$ preconditions for $\mathrm{t} 4$ transition. As a result, the $\mathrm{P} 10=1, \mathrm{P} 21=1, \mathrm{P} 40=1$ post-conditions are formed relative to the occurred event implemented by the actions of $\mathrm{t} 1$ transition and the $\mathrm{P} 50=1, \mathrm{P} 60=1, \mathrm{P} 71=1$ postconditions relative to the $\mathrm{t} 4$ transition. The formed post-conditions of the events presented are not preconditions of any other events, since logical descriptions of these preconditions with relation to the possible further $\mathrm{t} 2$ and $\mathrm{t} 5$ events are false:

$$
\begin{gathered}
\mathrm{t} 2=(\mathrm{P} 30=1) \cdot(\mathrm{P} 21=1) \cdot(\mathrm{P} 51=0)=0 \text { and } \\
\mathrm{t} 5=(\mathrm{P} 80=1) \cdot(\mathrm{P} 71=1) \cdot(\mathrm{P} 41=0)=0 .
\end{gathered}
$$




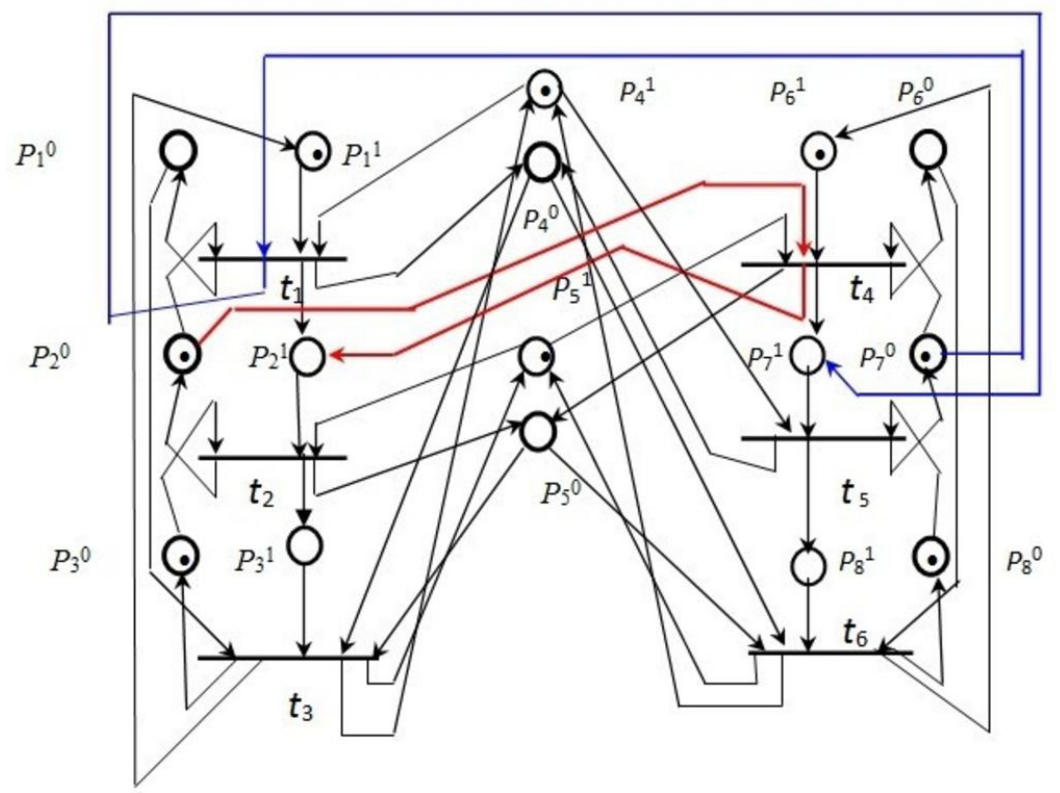

Fig. 1. Modeling of the distribution of resources with the possibility of occurrence of deadlocks, without regard to the coloured links and with using the dual Petri net.

As a result, there is a dead end occurring.

Thus, if a $t 4$ negative event occurs after the event represented by the $\mathrm{t} 1$ transition, this leads to a dead end, or if a $t 1$ negative event occurs after the $t 4$ event, it also leads the process to a dead end.

\section{Resources modelling}

In process of implementing the parallel and asynchronous processes in various fields of science and technology for modeling the behavior of modern developing information systems, people are based in most cases on artificial intelligence. Such systems use the incomplete knowledge of the test objects and the expert point of view is used in the designing process. The preliminary modeling with distributed processing of information is used to examine the more complete characteristics of such systems. Also, one of the convenient means of modeling is Petri nets, representing as a whole the functioning of software and hardware means and the corresponding activity of the system itself, including interaction with people.

An important characteristic of Petri nets is the presence of a mathematical tool that allows analyzing models based on basis of matrix equations. However, Petri nets have a limitation on modeling, namely, the inability to check a particular position for a zero marking. In addition, there are can be the limitations on the modeling power and the resolution power that Petri nets are trying to overcome. The modeling power characterizes the ability of models to represent the complex systems that are close to Turing machines with regard to flexibility, and the resolution power characterizes the possibility of performing a formal analysis of the resolutions of Petri nets. Also in some models there is occurring of a dissonance, because of the simultaneous occurrence of a direct and inverse state. 
The data of artificial intelligence, which are recorded in the expert system, can be either of clear structure or fuzzy structure. Clear rules and knowledge are represented as fuzzy ones with values close to final values. Thus, the expert system takes the following form:

Let us represent the compositional deriving rules for the machine of fuzzy deriving in the expert system, in the following form:

$$
\mathrm{F} \rightarrow \mathrm{G} ; \mathrm{F}^{\prime} / \mathrm{G}^{\prime} .
$$

The $\mathrm{G}^{\prime}$ deriving is determined from the «min-max» convolution of the F` fuzzy set and the $\mathrm{R}(5),(6)$ ratio, where:

$$
\begin{gathered}
\mathrm{R}=\mathrm{F} \times \mathrm{G}[8] ; \\
\mathrm{U}=\{\mathrm{u} 1, \mathrm{u} 2, \ldots, \mathrm{um}\} ; \mathrm{V}=\{\mathrm{v} 1, \mathrm{v} 2, \ldots, \mathrm{vm}\} \\
\mathrm{F}, \mathrm{F}^{\prime} \subset \mathrm{U} ; \mathrm{G}, \mathrm{G}^{\prime} \subset \mathrm{V} .
\end{gathered}
$$

As the result:

$$
\mathrm{Gi}=\max \{\min (\mu(\mathrm{fi}), \min ((\text { ui }),(\mathrm{vi}))), \min (\mu(\mathrm{fi}+1), \min ((u \mathrm{i}+1),(\mathrm{vi}+1)))\} ;
$$

$\mathrm{Gi}^{\prime}=\min \{\max \mu(\mathrm{fi}), \max (($ ui),(vi))), $\max (\mu(\mathrm{fi}+1), \max ((u i+1),(v i+1)))\}$.

Let us consider separately the «max» and «min» elements for building the dual Petri net of fuzzy deriving of knowledge (Fig. 2).

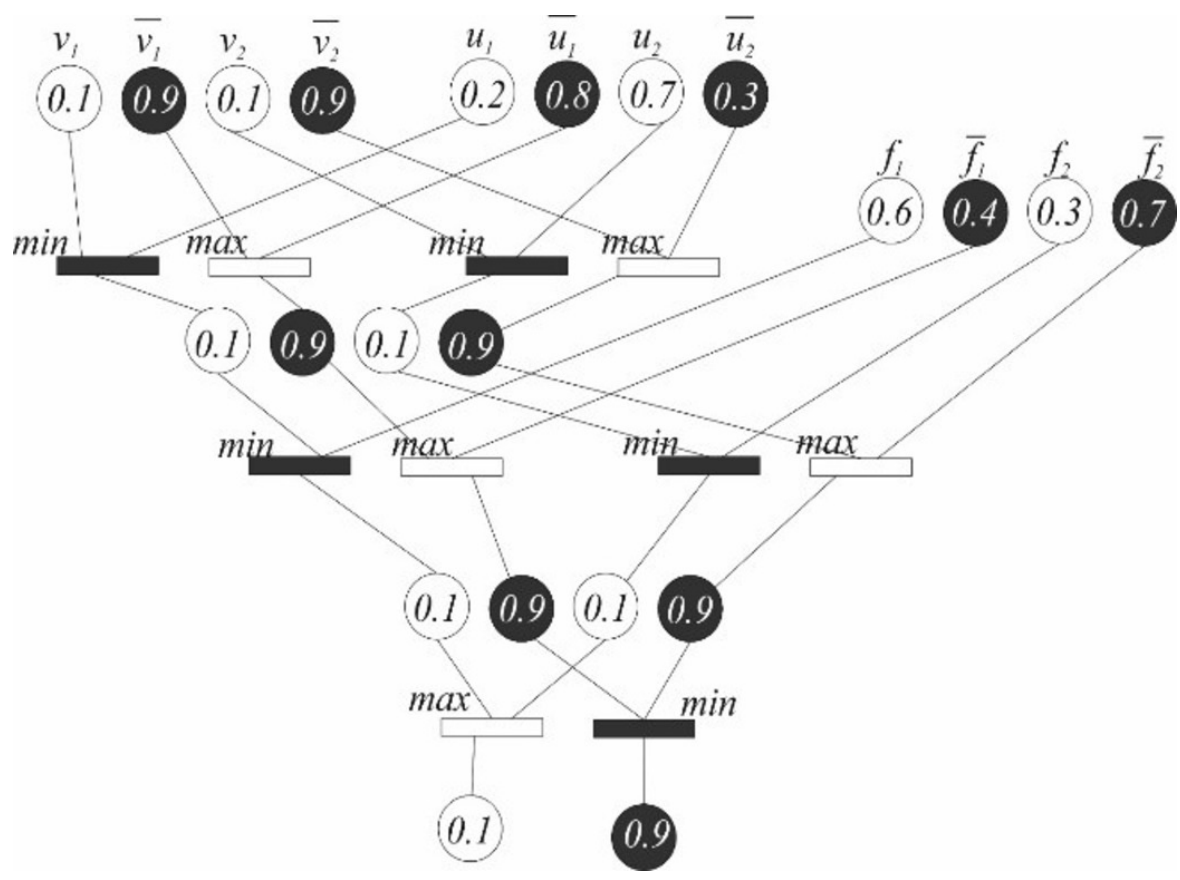

Fig. 2. Dual Petri net of fuzzy deriving of knowledge in the expert system.

From the resulting expression, we can represent the deriving branch corresponding to one of the functions of membership of the derived knowledge, in the form of a fuzzy deriving of the dual Petri net (Fig. 2). To change the markup of the Petri net with fuzzy deriving of knowledge, it is necessary to change the input positions of the marked membership functions of the linguistic variables from the segment $[0,1]$, by means of 
normalization, to the integer segment $[0,10]$ or $[0,100]$, depending on the digits capacity used. The integer markups of the output positions resulting from the deriving, using the reverse operation, is converted to fractional values corresponding to the membership functions of the derived knowledge.

$\mu(\mathrm{g} 1)$ here is an element of the matrix. $\mid \mu(\mathrm{g} 1), \mu(\mathrm{g} 2), \ldots,) \mu(\mathrm{gm}) \mid$ is the membership functions of the $\mathrm{G}^{\prime}$ deriving knowledge.

On basis of analyzing the results obtained in the modeling, we can say that the highest value and the lowest value of the initial marking is the result of modeling, an along with this, the minimum value is assigned to the value of the direct result, in the other case the result is the primary conjunctive interaction of the elements.

\section{Conclusions (Findings)}

Dual network Petri allows organizing the exclusion of the occurrence of negative events by the implementation of additional links, which makes it possible to consider the dead end states during the modeling of objects.

The structure of the fuzzy dual Petri net is implemented in accordance with the composite rule, which involves the use of transitions of the maximum and minimum values corresponding to the logical calculations of the conjunction and disjunction. The transition from classical Petri nets to the fuzzy dual Petri nets, helps to implement the fuzzy knowledge of logical deriving. Expert systems with fuzzy logic can solve the problem of analyzing the productive processes consisting of normalized and inverse positions. This property gives an opportunity to obtain new knowledge and solve the problem of building of more reliable expert systems.

\section{References}

1. V. E. Kotov, Petri nets (Nauka, Leningrad, 1984)

2. A. A. Leskin, P. A. Maltsev, A.M. Spiridonov, Petri nets in modeling and control (Nauka, Leningrad, 1989)

3. J. Peterson, Theory of Petri nets and systems modeling (Mir, Moscow, 1984)

4. Dm. V. Fatkhi, D. V. Fatkhi, Proceedings of the XXII International Scientific Conference, 5 (2009)

5. Dm. V. Fatkhi, V. A. Fathkhi, D. V. Fatkhi, Information security of regions. Research and practice journal, 2 (7), 69 (2010)

6. A. P. Ganzhur, M. A. Ganzhur, Dual Petri net (System analysis, management and processing of information / Don State Technical University, Rostov-on-Don, 2013)

7. M. A. Ganzhur, A.P. Ganzhur, V.A. Fatkhi, Proceedings of the First Research and Practice Conference, 16 (2014)

8. M. A. Ganzhur, D. V Fatkhi., A. P. Ganzhur, Proceedings of the VII International Workshop, 84 (2016) 